\title{
In Situ Monitoring of Silicon Membrane Thickness during Wet Etching using a Surface Acoustic Wave Sensor
}

\author{
Chi-Yuan LeE*, Ying-Chou Cheng, Yung-Yu ChEN ${ }^{1}$, Pei-Zen ChAng $^{1}$, Tsung-Tsong Wu ${ }^{1}$, \\ Ping-Hei Chen, Wen-Jong CHEN ${ }^{1}$ and Shih-Yung PAO ${ }^{1}$ \\ Department of Mechanical Engineering, National Taiwan University, Taipei 10617 Taiwan, R.O.C. \\ ${ }^{1}$ Institute of Applied Mechanics, National Taiwan University, Taipei 10617, Taiwan, R.O.C.
}

(Received October 12, 2003; accepted February 25, 2004; published June 9, 2004)

In this paper, we propose a novel method using the surface acoustic wave (SAW) sensor for monitoring in situ the thickness of a silicon membrane during wet etching. Similar to pressure sensors and accelerometers, some micro-electro-mechanical systems (MEMS) devices require the thickness of silicon membranes to be precisely known. Precisely controlling the thickness of a silicon membrane during wet etching is important, because it strongly influences post-processing and device performance. Furthermore, the proposed surface acoustic wave sensor enables the thickness of a silicon membrane, from $50 \mu \mathrm{m}$ to $80 \mu \mathrm{m}$, to be monitored in situ. In summary, the proposed method for measuring the thickness of a silicon membrane in real time from $50 \mu \mathrm{m}$ to $80 \mu \mathrm{m}$, is highly accurate, is simple to implement and can be used for mass production. The principles of the method, detailed process flows, the set up for measuring thickness and simulation and experimental results are all discussed. The theoretical and measured values differ by an error of less than $2 \mu \mathrm{m}$; thus the experimental and theoretical values correlate well with each other. [DOI: 10.1143/JJAP.43.3611]

KEYWORDS: SAW sensor, MEMS, in situ monitoring

\section{Introduction}

Anisotropic chemical wet etching is one of the key technologies for fabricating microstructures on a single crystal silicon wafer. ${ }^{1)}$ Similar to pressure sensors and accelerometers, some micro- electro-mechanical systems (MEMS) devices require the thickness of silicon membranes to be precisely known. Precisely controlling the thickness of a silicon membrane during wet etching is crucial, because the thickness strongly affects post-processing and device performance. Silicon has been thinned using various techniques, including chemical wet etching, ${ }^{2)}$ dry etching ${ }^{3)}$ and mechanical polishing. Chemical wet etching using many anisotropic etchants, including potassium hydroxide $(\mathrm{KOH})$, tetramethyl ammonium hydroxide (TMAH) and ethylenediamine-pyrocatechol (EDP), has been effectively implemented in mass production, ${ }^{4)}$ while dry etching precisely controls the thickness of the silicon membrane. The advantages and disadvantages of wet and dry etching are well known. ${ }^{2}$ )

A novel method, which differs from that of any previous work on etch-stop techniques, ${ }^{5-8)}$ develops a novel method in situ for monitoring the thickness of a silicon membrane from $50 \mu \mathrm{m}$ to $80 \mu \mathrm{m}$ with high accuracy, a simple setup and can be used for mass production. The principles of the method, detailed process flows, the set up for taking measurements and simulation and experimental results are discussed.

\section{Methodology}

2.1 Surface waves that propagate in a piezoelectric film based on a multi layer structure loaded with viscous liquid

The eight-dimensional matrix formalism has been derived and employed in the propagation of surface waves in piezoelectric film based on a multi layered structure, loaded

*Present address: Institute of Applied Mechanics (Lab. 433), National Taiwan University, 1, Sec. 4, Roosevelt Rd., 10617, Taipei, Taiwan. E-mail address: leecyu@mems.iam.ntu.edu.tw with viscous liquid. $\left.{ }^{9}{ }^{10}\right)$ This formulation derives the dispersion equation of surface waves in such a structure from continuity conditions at the solid-liquid interface. The size of the matrix in the computation is independent of the number of layers. This characteristic is helpful in analyzing the behaviors of waves in a multi layered structure.

Consider a piezoelectric film based on a silicon substrate loaded with viscous liquid in the half-space, where the interface is at $z=H$, as shown in Fig. 1. According to the eight-dimensional matrix formalism, at the interface, the relationship between the generalized traction vector $\boldsymbol{T}$ and the generalized velocity vector $\boldsymbol{V}$ is expressed as ${ }^{11)}$

$$
\boldsymbol{T}\left(H^{-}\right)=\boldsymbol{G} \boldsymbol{V}\left(H^{-}\right)
$$

where $\boldsymbol{G}$ is the impedance tensor, and $H$ is the thickness of a multi layered structure. In the viscous liquid half-space, since only up-going waves exist, the global impedance is

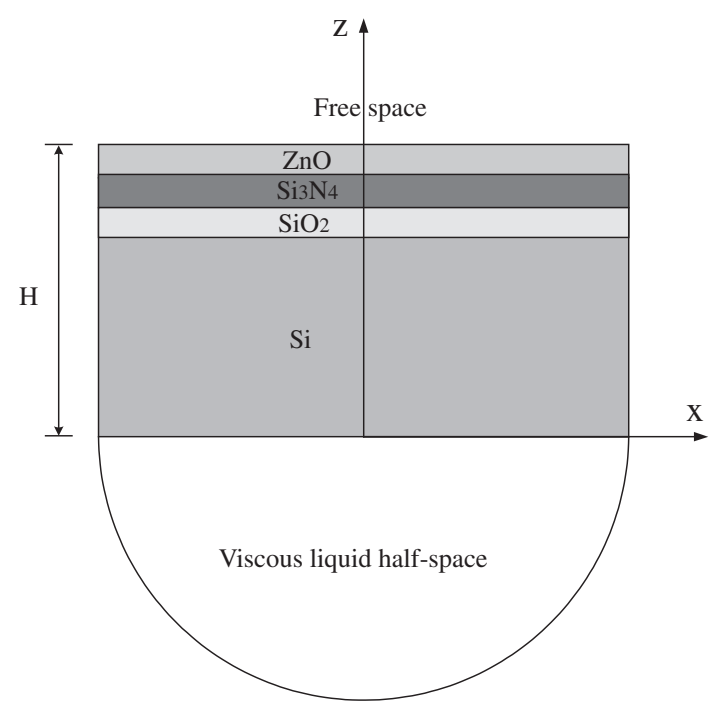

Fig. 1. Configuration of a piezoelectric film based on a silicon substrate loaded with viscous liquid in the half-space. 
equivalent to the up-going wave impedance $Z_{1}$. Consequently, the corresponding relationship at the boundary of the viscous liquid is given by

$$
\boldsymbol{T}_{1}\left(H^{+}\right)=Z_{1} V_{1}\left(H^{+}\right)
$$

At the liquid-solid interface, the stress, the particle velocity, the electric potential and the electric displacement must satisfy the continuity condition:

$$
\boldsymbol{T}\left(H^{-}\right)=\boldsymbol{T}_{1}\left(H^{+}\right), \quad \boldsymbol{V}\left(H^{-}\right)=\boldsymbol{V}_{1}\left(H^{+}\right)=\boldsymbol{V}(H)
$$

From eqs. (1), (2) and (3), we obtain

$$
\left(\boldsymbol{G}-\boldsymbol{Z}_{1}\right) \boldsymbol{V}(H)=0
$$

The non trivial solution of the generalized velocity vector $\boldsymbol{V}(H)$ exists only if

$$
\operatorname{det}\left(\boldsymbol{G}-\boldsymbol{Z}_{1}\right)=0
$$

Equation (5) is the also called the dispersion equation for the propagation of surface waves in a piezoelectric film based on a silicon substrate loaded with viscous liquid half-space.

On the basis of the foregoing formulation, the dispersion characteristics of the viscous-liquid-loaded $\mathrm{Si}(296.5 \mu \mathrm{m}) /$ $\mathrm{SiO}_{2}(1000 \AA) / \mathrm{Si}_{3} \mathrm{~N}_{4}(1500 \AA) / \mathrm{ZnO}(14180 \AA)$ multi layered system are studied. The epitaxial relationships are that the caxis of zinc oxide $(\mathrm{ZnO})$ is parallel to the surface of the (100) silicon substrate, and the Rayleigh wave propagates along the $\mathrm{X}$-axis of the $\mathrm{ZnO}$ film and in the $\langle 100\rangle$ direction of the silicon substrate. The relevant constants utilized in the calculations are adopted from ref. 12. Figure 2 shows a simulation result, which is phase velocity with respect to the silicon thickness. According to the simulation result, the phase velocity approaches the velocity of the Rayleigh wave of silicon as the thickness of the silicon increases. When the thickness of the silicon is less than the wavelength of the surface wave, the surface acoustic wave and the bulk wave combine into a plate wave, whose phase velocity varies with the thickness of the silicon substrate. The simulation results indicate that the attenuation increases as the thickness of the silicon membrane decreases, as shown in Fig. 3. The attenuation of surface acoustic wave has minimal effect on

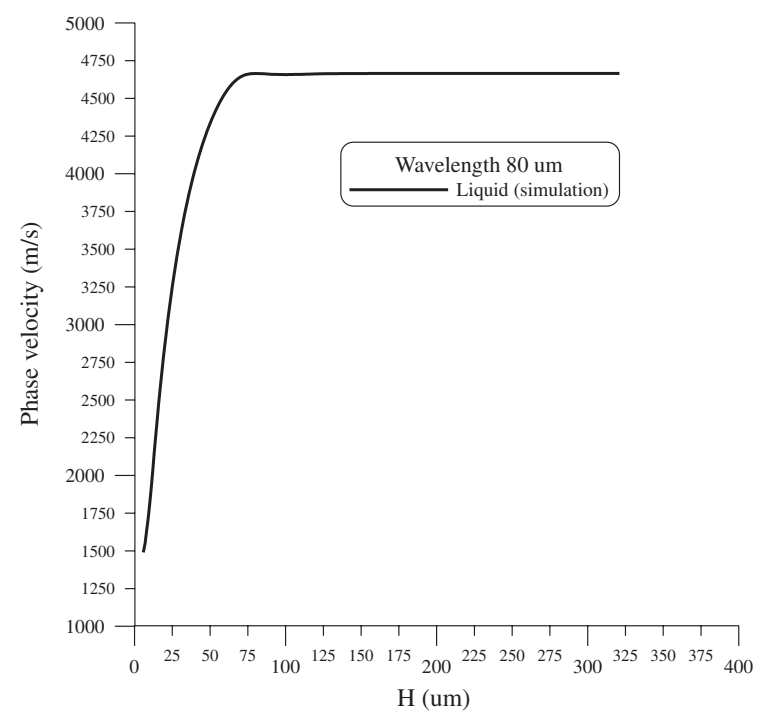

Fig. 2. Dispersion curve of a viscous-liquid-loaded silicon substrate.

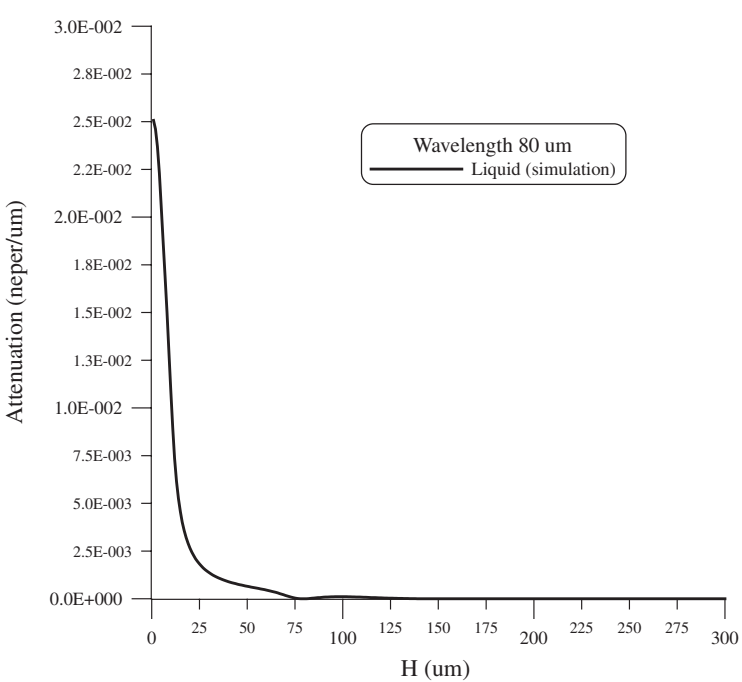

Fig. 3. Attenuation of surface acoustic wave of a viscous-liquid-loaded silicon substrate.

the determination of silicon membrane thickness if the membrane thickness is greater than $75 \mu \mathrm{m}$. The cause for the attenuation is mainly due to the energy leakage into the liquid half-space.

\subsection{Design of surface acoustic wave sensor}

A surface acoustic wave (SAW), also called the Rayleigh wave, is essentially a coupling between longitudinal and shear waves. The energy carried by the SAW is confined near the surface. An associated electrostatic wave exists for a SAW on a piezoelectric substrate, permitting electroacoustic coupling via a transducer. ${ }^{13)} \mathrm{A} \mathrm{ZnO}$ film has widely played an important role in the field of micro-electro-mechanical systems (MEMS), both in bulk micromachining, and in surface micromachining, because it has a high coupling coefficient, a strong piezoelectric effect, good piezoelectric performance and can be readily sputtered as an oriented crystalline composite on a wide variety of substrates. ${ }^{14)}$ Hence, $\mathrm{ZnO}$ was chosen as a piezoelectric film layer herein. A surface acoustic wave sensor was fabricated on a $\mathrm{ZnO}$ layer based on a double-polished (100) silicon substrate, using the two ports of split-electrode interdigital transducers (IDT), as shown in Fig. 4. A $\mathrm{ZnO}$ thin film was deposited using sputtering technology. Figure 5 displays the X-ray diffraction (XRD) scans of a $\mathrm{ZnO}$ film, which reveal that a $\mathrm{ZnO}$ film exhibits a good c-axis orientation. Figure 6 presents the scanning electron microscope (SEM) images of optimized 1.418- $\mu \mathrm{m}$-thick $\mathrm{ZnO}$ film. The top view in Fig. 6(a) shows the uniformity and compactness of the grains. The cross-sectional view in Fig. 6(b) indicates that $\mathrm{ZnO}$

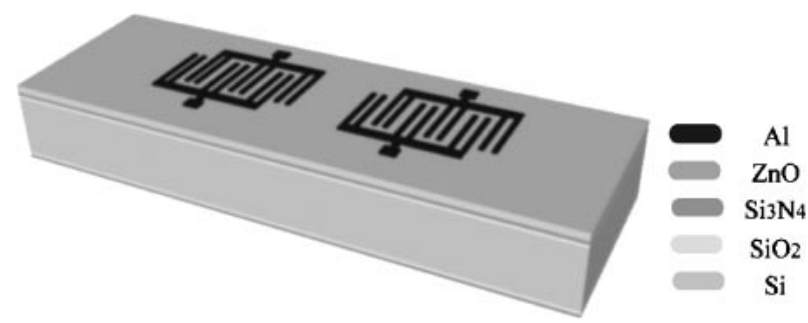

Fig. 4. Schematic diagram of a surface acoustic wave sensor. 


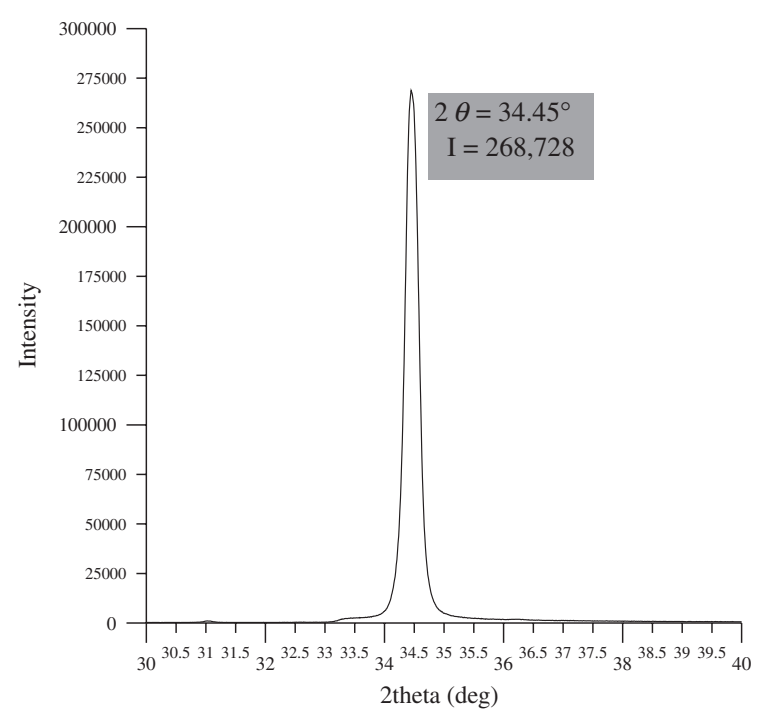

Fig. 5. X-ray diffraction scans of a $\mathrm{ZnO}$ film.

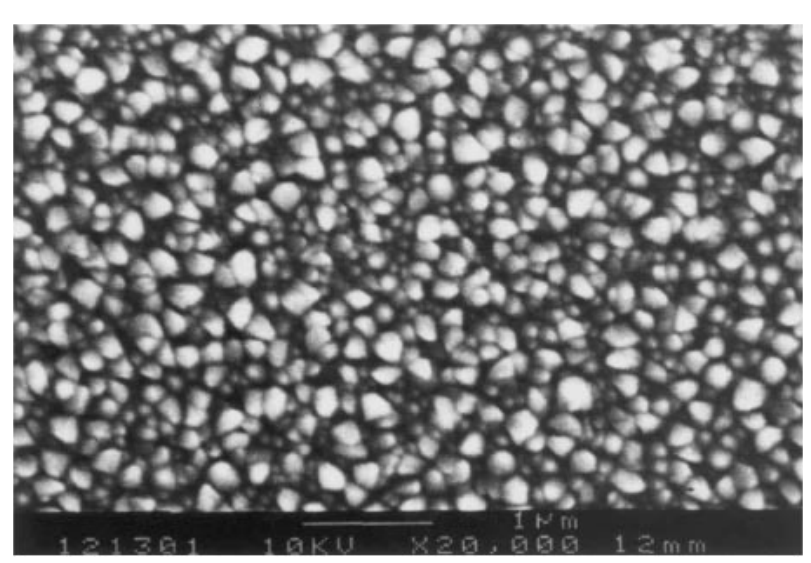

(a) Top view

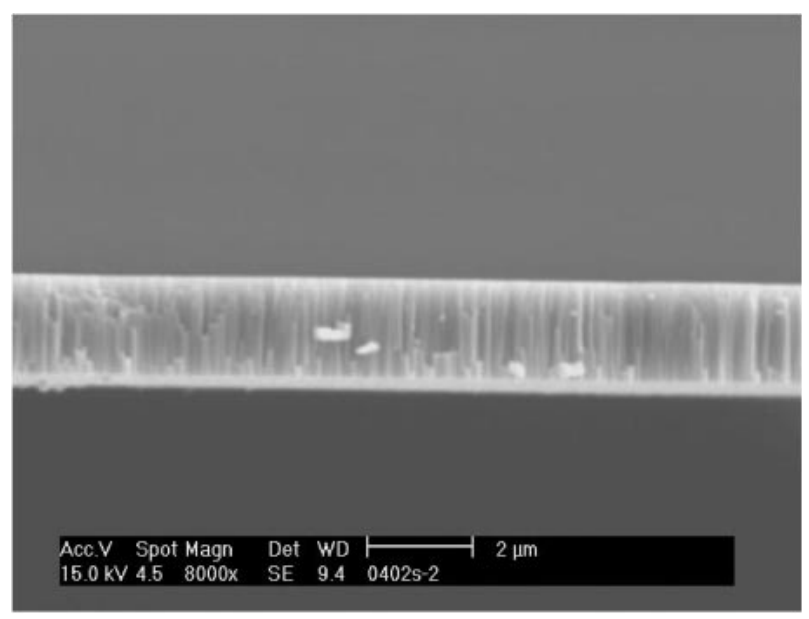

(b) Cross-sectional view

Fig. 6. SEM images of $\mathrm{ZnO}$ films.

columnar grains are clear and vertical to the substrate with a slight angle, indicating that $\mathrm{ZnO}$ grains have good orientation. The piezoelectric effect is such that, when the AC voltage was applied to the input IDT, and signal voltage

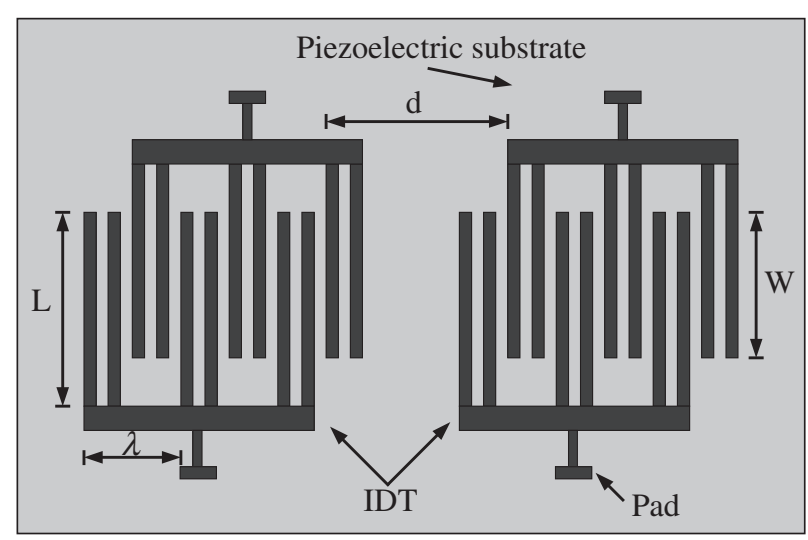

Fig. 7. Schematic configuration of the IDT.

Table I. IDT design parameters.

\begin{tabular}{lc}
\hline Interdigital transducer (IDT) & Value of parameter \\
\hline Periodicity of IDT $(\lambda)$ & $80 \mu \mathrm{m}$ \\
Length of the IDT fingers $(L)$ & $3360 \mu \mathrm{m}$ \\
$\quad \begin{array}{l}\text { Length of overlap of the IDT } \\
\quad \text { fingers }(W)\end{array}$ & $3040 \mu \mathrm{m}$ \\
$\quad \begin{array}{l}\text { Length of the path of acoustic } \\
\quad \text { propagation }(d)\end{array}$ & $1520 \mu \mathrm{m}$ \\
$\quad \begin{array}{l}\text { Numbers of pairs }(N) \\
\text { Area of pad }\end{array}$ & 95 \\
\hline
\end{tabular}

variations were subsequently converted into a mechanical acoustic wave, the other IDT acted as an output receiver to convert mechanical SAW vibrations back into an output voltage. The output voltage or the wave velocity changes with the thickness of a silicon substrate.

Figure 7 shows a configuration of the IDT, where $\lambda$ is the periodicity of IDT (wavelength of the surface acoustic wave); $L$ is the length of the IDT fingers; $W$ is the acoustic aperture (length of overlap of the IDT fingers); $d$ is the length of the path of acoustic propagation and $N$ is the number of pairs. In the design of IDT, a number of parameters have to be specified. The spacing and the electrode width will determine the frequency response of the transducer. In the proposed design, a split-electrode IDT was selected, because the problem that results from finger reflections can be greatly diminished using a $\lambda / 8$ finger width instead of a $\lambda / 4$ finger width, such that the SAW reflections from each split-electrode pair cancel out at the center frequency, rather than accumulate as in the case of the single-electrode IDT. ${ }^{15)}$ Table I lists the parameters of the IDT design used in this experiment.

\section{Fabrication}

This surface acoustic wave sensor was fabricated by the process shown in Fig. 8. The process was begun with a 296.5- $\mu$ m-thick 100-mm-diameter double-polished (100) silicon wafer. First, a silicon dioxide $\left(\mathrm{SiO}_{2}\right)$ film, around $1000 \AA$ thick, was deposited, before a low-pressure chemical vapor deposition (LPCVD) silicon nitride $\left(\mathrm{Si}_{3} \mathrm{~N}_{4}\right)$ film with a thickness of $1500 \AA$ was deposited, to reduce the stress, as shown in Fig. 8(a). The LPCVD $\mathrm{Si}_{3} \mathrm{~N}_{4}$ film was used as an etching mask during wet etching in $\mathrm{KOH}$ solution. Then, on 


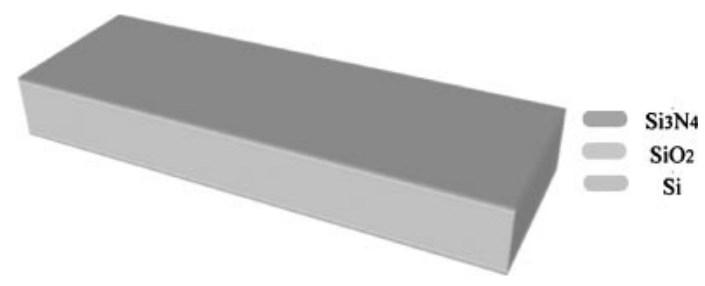

(a)

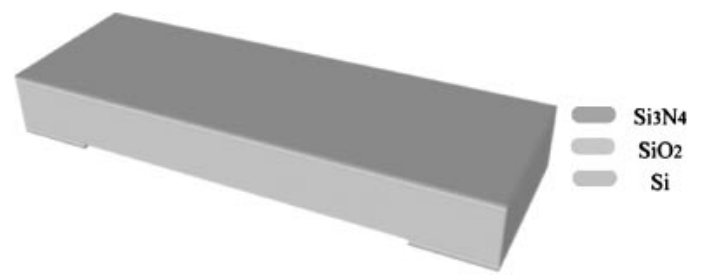

(b)

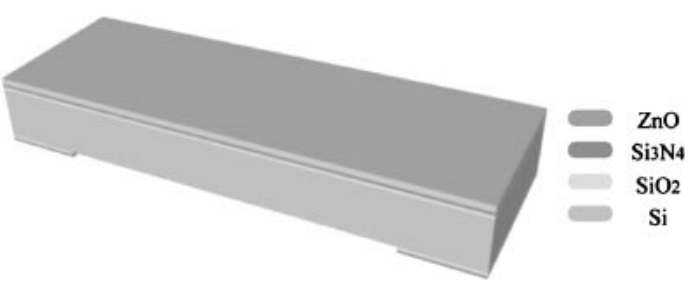

(c)

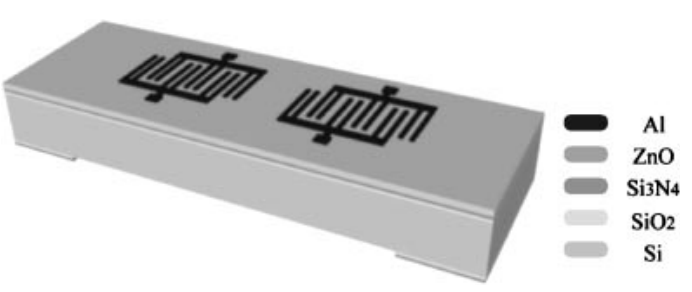

(d)

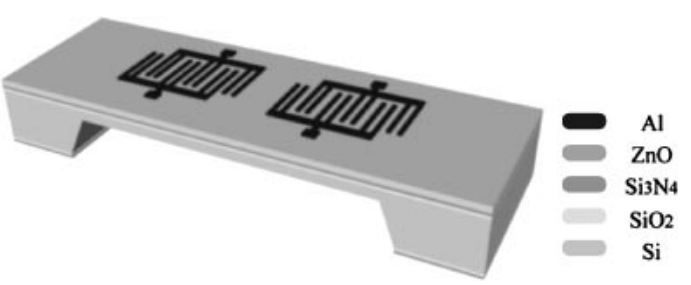

(e)

Fig. 8. Process flow of a surface acoustic wave sensor. (a) Growing a silicon dioxide film and an LPCVD silicon nitride film. (b) Defining the pattern on the rear side of the etching hole. (c) Sputtering a piezoelectric $\mathrm{ZnO}$ film. (d) Evaporating $\mathrm{Al}$ film and defining the pattern of IDTs at 45 degrees. (e) $\mathrm{KOH}$ etching to form a silicon membrane.

the rear side of the silicon substrate, the Shipley S1813 photoresist was coated using a spinner that rotated at a rate of $1000 \mathrm{rpm}$ for $10 \mathrm{~s}$, and then at $4000 \mathrm{rpm}$ for $35 \mathrm{~s}$. The coated substrate was then soft-baked at $90^{\circ} \mathrm{C}$ for $90 \mathrm{~s}$. The etching-hole area pattern was exposed onto the photoresistcoated substrate surface using ultraviolet light. Reactive ion etching (RIE) was applied to open the rear side of the etching hole, as shown in Fig. 8(b). A 1.418- $\mu$ m-thick $\mathrm{ZnO}$ film was sputtered using an RF magnetron sputtering system, as shown in Fig. 8(c). A ZnO film was prepared using an RF magnetron sputtering system, under the conditions described
Table II. Sputtering conditions.

\begin{tabular}{lc}
\hline \multicolumn{1}{c}{ Target } & $\mathrm{ZnO}, 4^{\prime \prime}$ diameter \\
\hline Gas $(\mathrm{sccm})$ & $\mathrm{Ar} / \mathrm{O}_{2}=1: 1$ \\
Deposition pressure $(\mathrm{Pa})$ & $9 \times 10^{-5}$ \\
RF power $(\mathrm{W})$ & 200 \\
Substrate temperature $\left({ }^{\circ} \mathrm{C}\right)$ & 215 \\
Sputtering rate $(\AA / \mathrm{hr})$ & 4988 \\
Working distance $(\mathrm{cm})$ & 13 \\
\hline
\end{tabular}

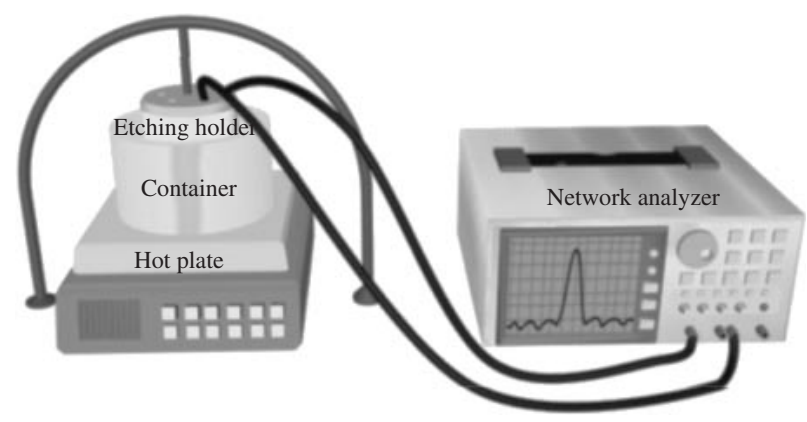

Fig. 9. Experimental setup of a surface acoustic wave sensor.

in Table II. IDTs were patterned using semiconductor photolithographic techniques, by which $\mathrm{Al}$ was deposited at a thickness at $950 \AA$ by thermal evaporation, as shown in Fig. 8(d). Finally, a silicon membrane was generated by soaking the rear side of the substrate with $73^{\circ} \mathrm{C}, 33 \mathrm{wt} . \%$ $\mathrm{KOH}$ solution, and the etching rate was $0.97 \mu \mathrm{m}$ per minute, as shown in Fig. 8(e).

\section{Results}

The experimental setup for measuring the frequency response and the insertion loss of a surface acoustic wave sensor, as illustrated in Fig. 9, includes an HP8714ES network analyzer and an etching holder. An HP8714ES network analyzer monitor was used for monitoring in real time the thickness of a silicon membrane as the target central frequency was shifted. When the central frequency shifts, the thickness of a silicon membrane can be determined on the basis of the amount of shift of the central frequency. The etching process automatically monitors the thickness of a silicon membrane as the frequency changes. First, an Alphastep 500 surface profiler was used to measure the initial total thickness of 296.5- $\mu$ m-thick silicon, as shown in Fig. 10(a). Then, the rear side of the silicon substrate was attached onto a PCB by alkali-resistant epoxy, as shown in Fig. 10(b). A rectangular hole with a dimension of $1.8 \mathrm{~mm}$ by $0.7 \mathrm{~mm}$ was cut off from the PCB. The rectangular hole serves as an open window for etching on the rear side of the silicon substrate. The Alpha-step 500 surface profiler was used to measure the thickness of 430.2- $\mu$ m-thick PCB, as shown in Fig. 10(c). The thickness of alkali-resistant epoxy was $141.02 \mu \mathrm{m}$. The hole is too deep to measure using the Alpha-step 500 surface profiles. Instead, an optical $\mu$ scan AF2000 with a $z$-axis resolution of $25 \mathrm{~nm}$ was used to measure the initial depth of the open window, which was $571.22 \mu \mathrm{m}$ thick, as shown in Fig. 10(d). Once the etching process on the silicon substrate by $\mathrm{KOH}$ solution through the open window takes place, the silicon substrate exposed to the etchant becomes thinner. 


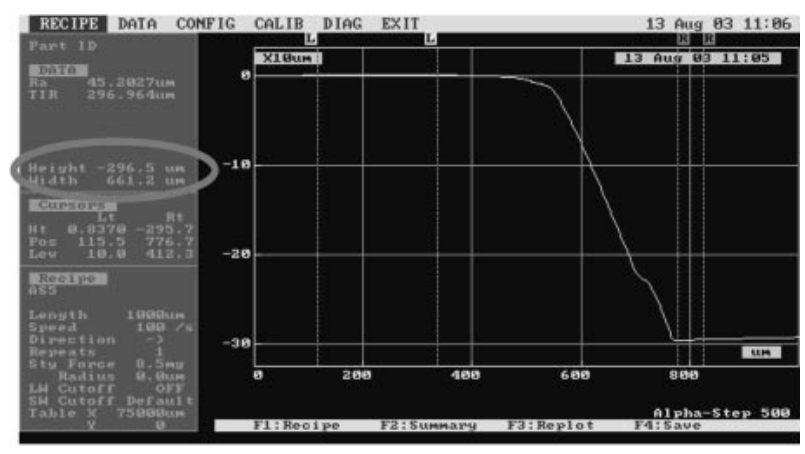

(a)

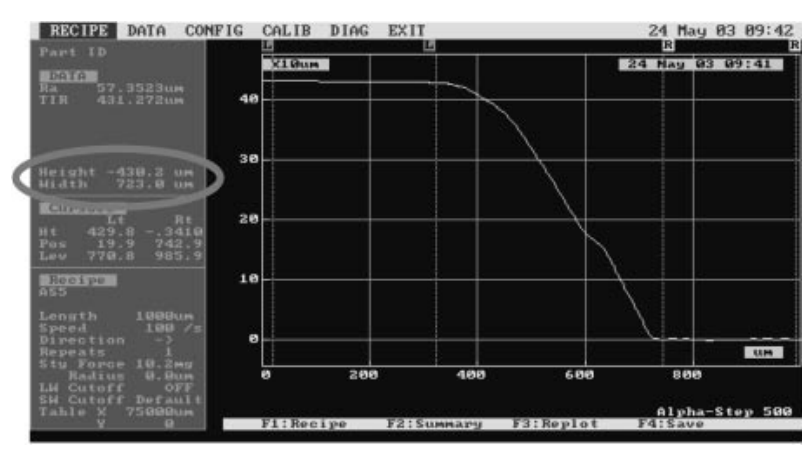

(c)

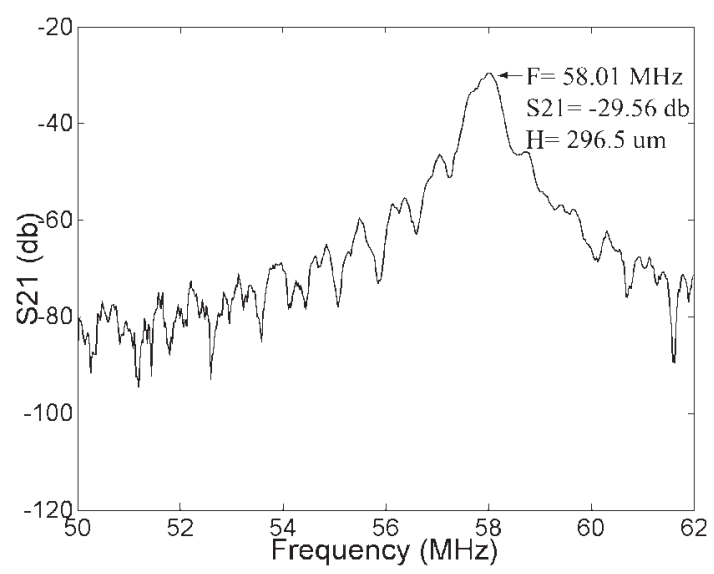

(e)

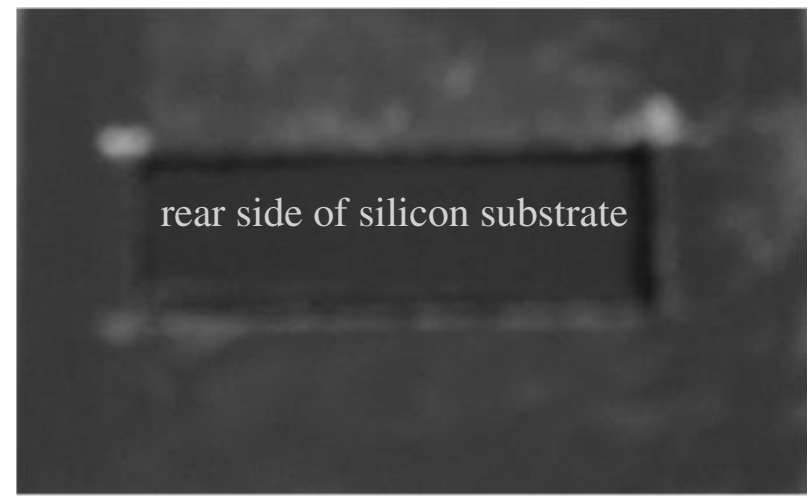

(b)

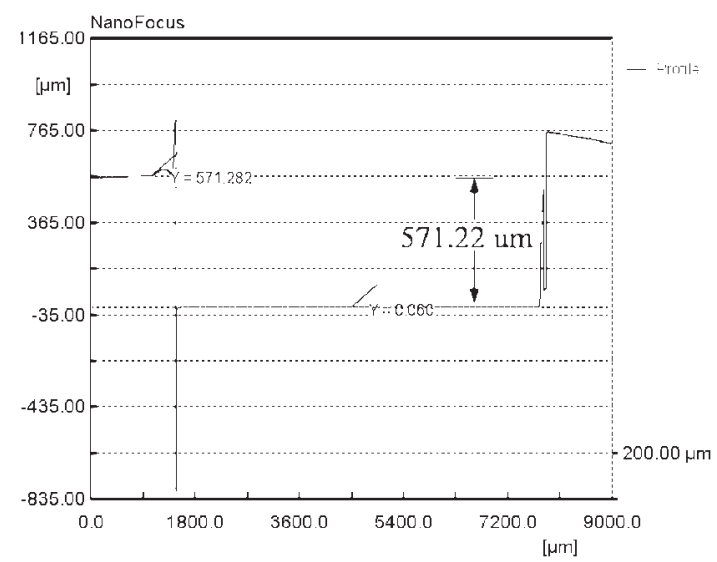

(d)

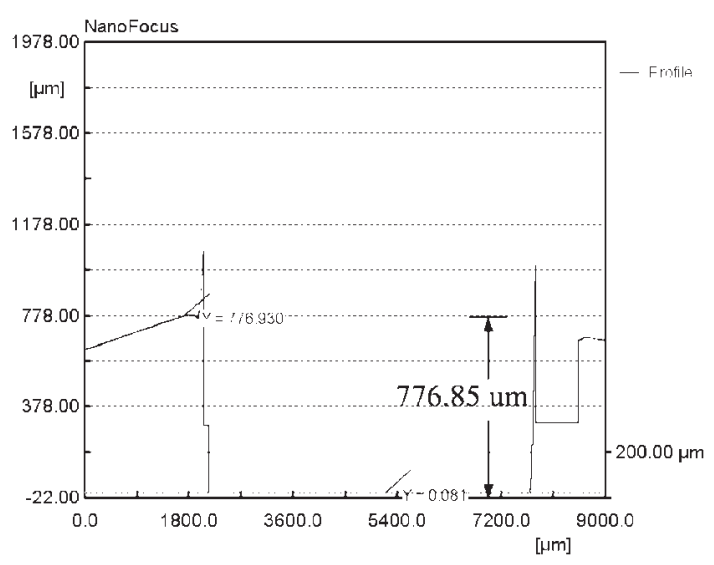

(f)

Continued on next page.

Fig. 10.

Consequently, the depth of the open window increases with etching time. The central frequency of the experimental unetched silicon substrate was $58.01 \mathrm{MHz}$, as shown in Fig. 10(e). After the first etching on the rear side of silicon, the depth of the open window was determined to be $776.85 \mu \mathrm{m}$ thick, as shown in Fig. 10(f). Therefore, the etching depth of the silicon substrate is $205.63 \mu \mathrm{m}$ after the first etching using the $\mathrm{KOH}$ solution. Now, the thickness of the silicon membrane can then be determined at $90.87 \mu \mathrm{m}$. An experimental result of the first etched central frequency was $58.01 \mathrm{MHz}$, as shown in Fig. $10(\mathrm{~g})$. Repeating these steps reveal that the depth of open window after the second etching process was determined to be $802.94 \mu \mathrm{m}$ thick, as shown in Fig. 10(h). An experimental result of the second etched central frequency was $57.5 \mathrm{MHz}$; the thickness of the silicon membrane was $64.78 \mu \mathrm{m}$, as shown in Fig. 10(i). The 


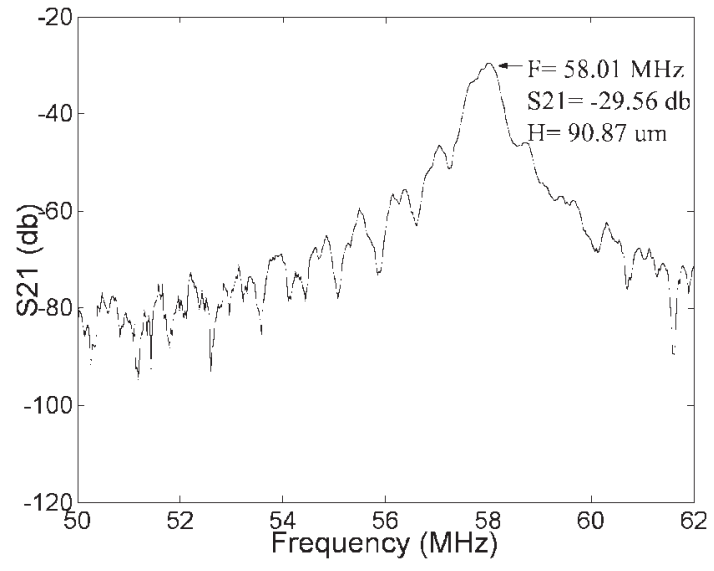

(g)

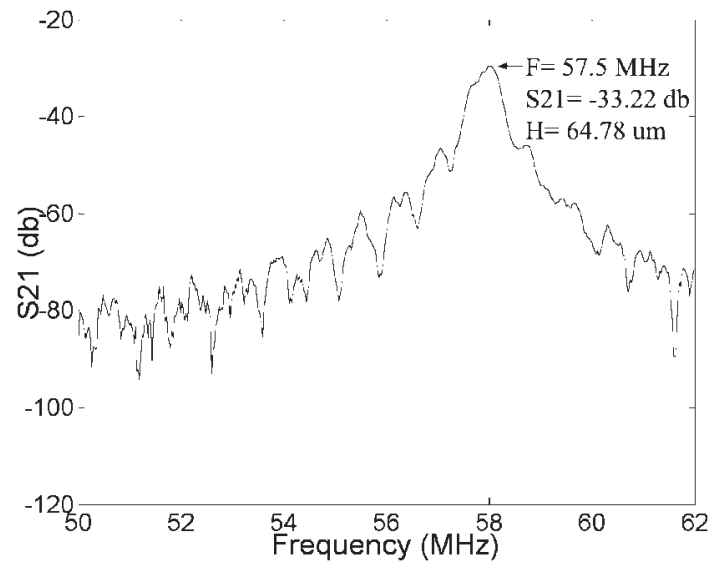

(i)

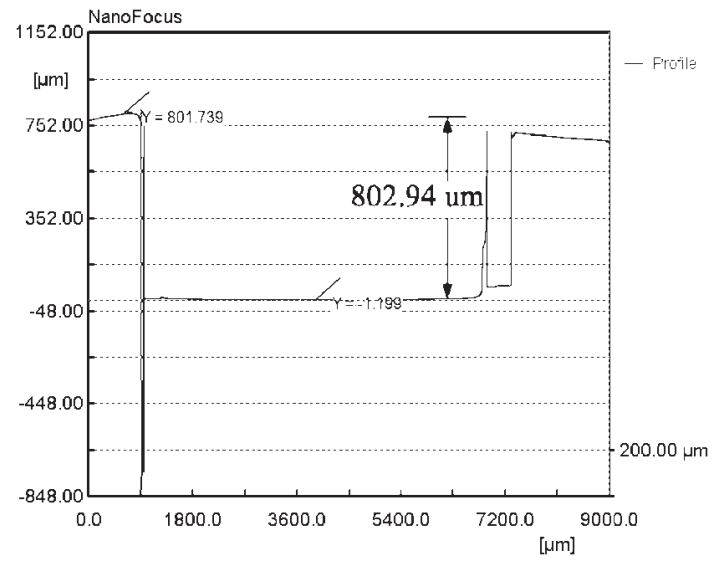

(h)

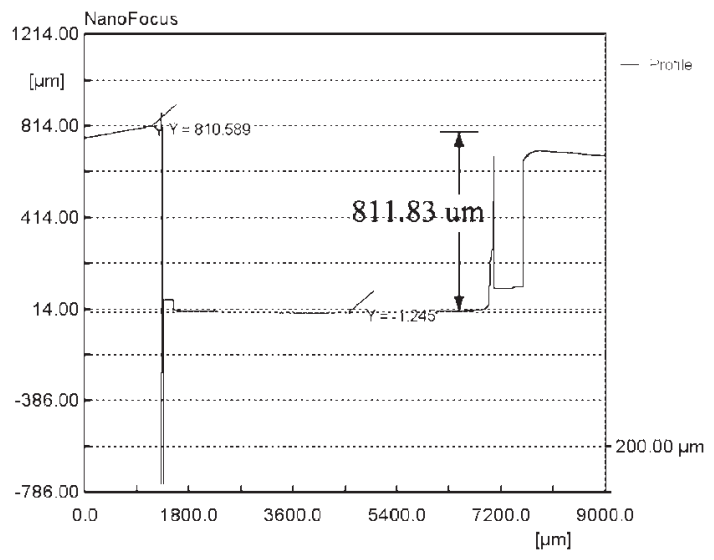

(j)

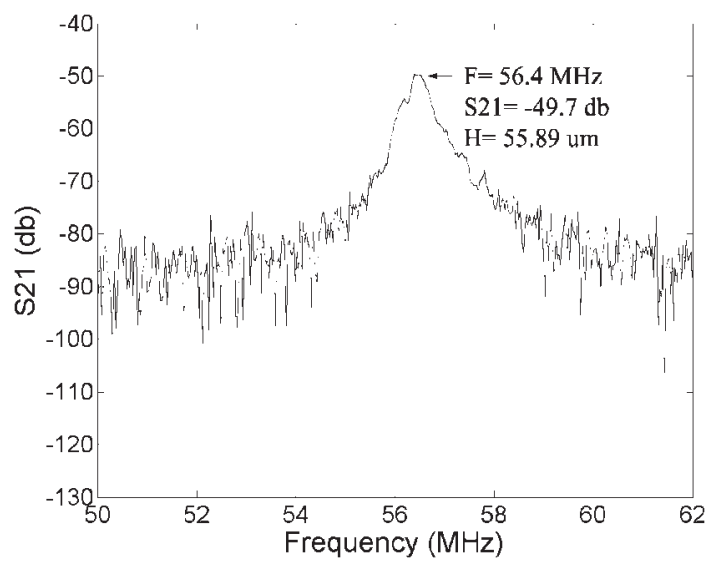

(k)

Fig. 10. Experimental results with unetched and etched for the central frequency versus insertion loss, and images of the thickness of silicon membrane obtained by $\mu$ scan AF2000. (a) Photograph of initial total thickness of 296.5- $\mu$ m-thick silicon, obtained by Alphastep 500 surface profiler. (b) Photograph of rear side of silicon substrate that was attached onto a PCB by alkali-resistant epoxy. (c) Photograph of thickness of 430.2- $\mu$ m-thick PCB, obtained by Alpha-step 500 surface profiler. (d) Photograph of initial depth of open window before etching, which was $571.22 \mu \mathrm{m}$ thick, obtained by $\mu$ scan AF2000. (e) Experimental results of unetched central frequency versus insertion loss, in which the central frequency was $58.01 \mathrm{MHz}$; the thickness of the silicon membrane was $296.5 \mu \mathrm{m}$. (f) Photograph of depth of open window after the first etching process, which was $776.85 \mu \mathrm{m}$ thick, obtained by $\mu$ scan AF2000. (g) Experimental results of first etched central frequency versus insertion loss, in which the central frequency was $58.01 \mathrm{MHz}$; the thickness of the silicon membrane was $90.87 \mu \mathrm{m}$. (h) Photograph of depth of open window after the second etching process, which was $802.94 \mu \mathrm{m}$ thick, obtained by $\mu$ scan AF2000. (i) Experimental results of second etched central frequency versus insertion loss, in which the central frequency was $57.5 \mathrm{MHz}$; the thickness of the silicon membrane was $64.78 \mu \mathrm{m}$. (j) Photograph of depth of open window after the third etching process, which was $811.83 \mu \mathrm{m}$ thick, obtained by $\mu$ scan AF2000. (k) Experimental results of third etched central frequency versus insertion loss, in which the central frequency was $56.4 \mathrm{MHz}$; the thickness of the silicon membrane was $55.89 \mu \mathrm{m}$. 


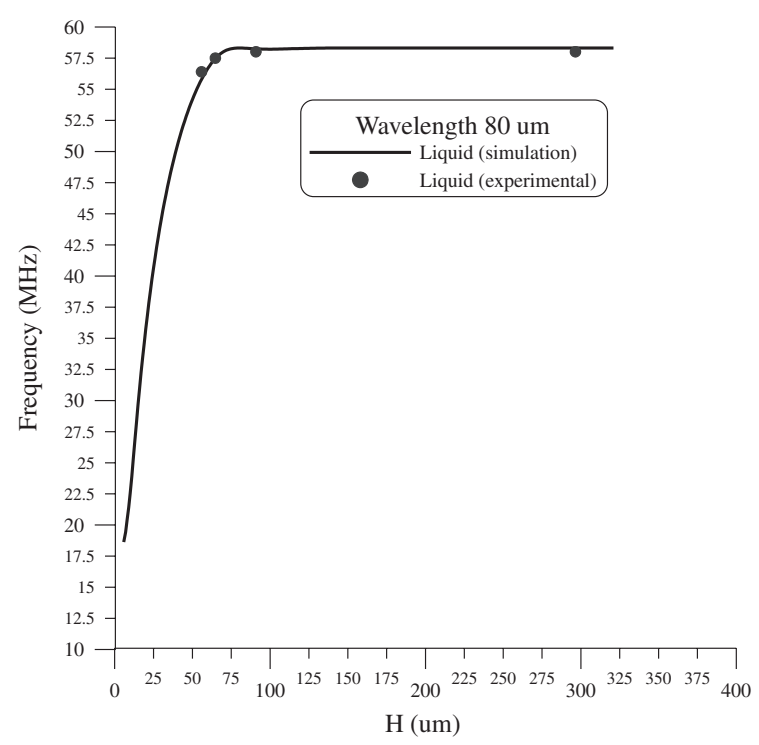

Fig. 11. Comparison of simulation and experimental results of thickness of silicon with respect to frequency.

depth of the open window after the third etching process was determined to be $811.83 \mu \mathrm{m}$ thick, as shown in Fig. 10(j). An experimental result of the third etched central frequency was $56.4 \mathrm{MHz}$; the thickness of the silicon membrane was $55.89 \mu \mathrm{m}$, as shown in Fig. 10(k). Figure 11 compares the simulation and experimental results of silicon thickness with respect to frequency. Figure 11 reveals that the error between the simulation and experimental results is less than $2 \mu \mathrm{m}$, implying close correspondence.

\section{Conclusions}

In this work, we presented a novel surface acoustic wave sensor for monitoring the thickness of a silicon membrane from $50 \mu \mathrm{m}$ to $80 \mu \mathrm{m}$ in real time during wet etching. The principles of the method, detailed process flows, the set up for measuring thickness and simulation and experimental results were all discussed. The theoretical and measured values differ by an error of less than $2 \mu \mathrm{m}$, thus the experimental and theoretical values correlate well with each other.

\section{Acknowledgements}

This work was accomplished with much needed support and the authors would like to thank Kai-Hsiang Yen, ShihYang Liu, Fu-Yuan Xiao, Chih-Wei Liu, Dr. Jen-Yi Chen, Jing-Hung Chiou, Tsung-Wei Huang, Chia-Hua Chu, X. Y. Wang of the Institute of Applied Mechanics, National Taiwan University, and Professors Lung-Jieh Yang, ChingLiang Dai and Chien-Liu Chang for their valuable advice and assistance in experiment. Finally, we would like to thank the NSC Northern Region MEMS Research Center for kindly making their research facilities completely available.

1) G. T. A. Kovacs, N. I. Maluf and K. E. Petersen: Proc. IEEE 86 (1998) 1536.

2) K. R. Williams and R. S. Muller: J. microelectromech. Syst. 5 (1996) 256.

3) J. Kiihamaki, H. Kattelus, J. Karttunen and S. Franssila: Sens. Actuat. 82 (2000) 234.

4) N. Fujitsuka, K. Hamaguchi, H. Funabashi, E. Kawasaki and T. Fukada: 12th Int. Conf. Solid State Sensors, Actuators and Microsystems, 2003, p. 1667.

5) K. Minami, H. Tosaka and M. Esashi: J. Micromech. Microeng. 5 (1995) 41

6) M. Madou: Fundamentals of Microfabrication (CRC Press, New York, 1997).

7) C. M. A. Ashruf, P. J. French, P. M. Sarro, P. M. M. C. Bressers and J. J. Kelly: Mechatronics 8 (1998) 595.

8) P. Z. Chang and L. J. Yang: J. Micromech. Microeng. 8 (1998) 182.

9) T. T. Wu and M. P. Chang: Jpn. J. Appl. Phys. 41 (2002) 5451.

10) T. T. Wu and T. Y. Wu: Transcations of the ASME 66 (2002) 262.

11) M. B. Braga: Ph.D. dissertation (Stanford University, Stanford, CA, 1990).

12) B. A. Auld: Acoustic Fields and Waves in Solid (R. E. Krieger, New York, 1990) Vol. I.

13) K. Uchino: Ferroelectric Devices (Marcel Dekker, New York, 2000).

14) T. Xu, G. Wu, G. Zhang and Y. Hao: Sens. Actuat. 104 (2003) 61.

15) C. K. Campbell: Surface Acoustic Wave Devices for Mobile and Wireless Communication (Academic Press, New York, 1998). 